\title{
Olfactory and Gustatory Alterations in Covid-19 Patients: A Tertiary Care Covid-19 Centre Inpatient Experience
}

\author{
Disha Koul $^{1}$ (D) Raies Ahmad Begh $^{1} \cdot$ Parmod Kalsotra $^{1}$
}

Received: 4 September 2020 / Accepted: 11 January 2021/Published online: 28 January 2021

(C) Association of Otolaryngologists of India 2021

\begin{abstract}
Corona virus disease 2019 (COVID-19) has emerged as a deadly pandemic throughout the world. Many case series from all over the globe and in India are detecting a very high frequency of chemo sensitive disorders in COVID-19. There is increasing evidence that olfactory and gustatory dysfunction can be present in COVID-19 patients. Olfactory and gustatory function was subjectively tested in 300 COVID-19 patients admitted at our hospital, Government Medical College, Jammu first through telephonic interviews followed by online questionnaire. Among our test population, 159 patients (53\%) reported olfactory and gustatory alterations within 5 days of testing positive for COVID-19. 52\% of those complaining olfactory dysfunction developed it before testing positive while $45 \%$ of those having gustatory dysfunction reported the same before testing positive for the disease. Olfactory and gustatory dysfunctions represent common clinical findings in COVID-19 patients. Otorhinolaryngologists and head-neck surgeons must by now keep this diagnostic option in mind when evaluating cases of nonspecific anosmia and ageusia that arose suddenly and are not associated with rhinitis/influenza symptoms.
\end{abstract}

Keywords COVID-19 - SARS-CoV-2 - Anosmia · Hyposmia · Ageusia .

Raies Ahmad Begh

raies987@gmail.com

Disha Koul

sdkoul@gmail.com

1 Department of ENT and Head and Neck Surgery, SMGS

Hospital, Government Medical College, Jammu,

Jammu and Kashmir 180 001, India
Olfactory and gustatory dysfunction (OGD) . Otorhinolaryngologists

\section{Introduction}

December 2019 marked the beginning of Corona virus disease 2019 (COVID-19) which emerged as a global pandemic starting from Wuhan, Hubei province, China and then taking over the world map [1-3]. On February 11, 2020 WHO and ICTV (International Committee on Taxonomy of Virus) announced Severe Acute Respiratory Syndrome Corona Virus 2 (SAR-CoV-2) as the name of previously called 2019-Novel Corona virus, which is responsible for COVID-19 disease [4] The name was chosen because the virus is phylogenetically related to corona virus (SARS-CoV) which was responsible for SARS outbreak in year 2003 [5].

In COVID-19 patients, main clinical features are fever and cough, associated with lymphocytopenia and groundglass opacity on chest computed tomography [3]. Besides, some patients may present with upper respiratory symptoms such as pharyngodynia, sore throat, nasal congestion, rhinorrhoea and olfaction alterations [6, 7]. Olfactory dysfunction (OD), including anosmia and hyposmia, manifests itself particularly prominently among these symptoms in COVID-19 patients [8].

Olfactory dysfunction in COVID-19 infection could be related to the involvement of the olfactory bulb or to peripheral damage of the olfactory receptor cells in the nasal neuroepithelium [9]. This assertion is based on the potential neurotropic features of SARS-CoV-2. In particular, it has been demonstrated in transgenic mice that after intranasal administration of SARS-CoV, the virus could penetrate into the brain through the olfactory bulb, leading 
to rapid trans neuronal spread [10]. It is also well recognized that alterations in the volume and composition of saliva can disturb taste sensation [11]. Previously, epithelial cells lining salivary gland ducts were found to be early target cells of SARS coronavirus infection in the upper respiratory tracts of rhesus macaques [12].

Multiple pieces of evidence confirmed that the nasal cavity is a vital area susceptible to SARS-CoV-2 infection. Viral loads in the patient's nasal cavity were higher than the viral loads in the pharynx, both symptomatic individuals and asymptomatic ones, hinting the nasal cavity as the first gateway for the initial infection [13]. The researchers investigated the expression of SARS-CoV-2 entry-associated genes, ACE2 and TMPRSS2, in single-cell RNA-sequencing datasets from different tissues in the human body [14]. Goblet cells and ciliated cells in the nasal mucosa may be the initial site of SARS-CoV-2 infection, implicating primary SARS-CoV-2 transmission is through infectious droplets [14]. Furthermore, SARS-CoV-2 was detected in the tears of COVID-19 patient and can cause nasal infection via the nasolacrimal duct $[14,15]$. Therefore, these findings could explain the highly infectious and highly pathogenic nature of COVID-19 [5].

\section{Material and methods}

The study was conducted on 300 patients admitted in our hospital, Government Medical College, Jammu from 15 May, 2020 to 15 August, 2020. Symptomatic patients aged 18 years and above with positive results on Reverse transcriptase polymerase chain reaction (RT-PCR) were included in the study.

Exclusion criteria:

Uncooperative patients,

Patients on assisted ventilation,

Patients with no symptoms who were kept quarantine in primary or secondary care quarantine centres,

Previous surgery or radiotherapy in the oral and nasal cavities,

Pre-existing manifestations of smell and taste alterations before epidemic, History of head neck trauma,

History of nasal surgeries, Allergic rhinitis, chronic rhinosinusitis, Nasal polyposis.

History of diabetes mellitus, psychiatric or neurological disorders.

Patients who died before prescribed duration of study. All 300 patients provided informed consent and participated in the study which was approved by the ethics committee of the institute. Patients were telephonically interviewed after five days of being diagnosed with the disease resolution of key symptoms (such as cough, fever, dyspnoea, headache, myalgia and arthralgia) and data was collected in standardized questionnaire proforma. Patients were followed up weekly for 4 weeks for symptom profile and clinical condition. Data was analysed statistically.

\section{Result}

In our study, out of total 300 patients, 222 were males and 78 were females. Majority ( $81 \%)$ of the patients belonged to the age group of 20-40 years with mean age group of 37 $( \pm 7)$ years (Fig. 1).

Out of total 300 patients, $60 \%$ presented with fever as predominant symptom, $51 \%$ presented with headache as predominant symptom, $48 \%$ presented with myalgias and arthralgias. Cough was the predominant symptom in $42 \%$ of patients, sore throat in $39 \%$ while abdominal symptoms were predominant in $38 \%$ of patients. $35 \%$ patients complained of smell disturbances, $18 \%$ patients presented with rhinorrhoea and $15 \%$ patients presented with taste disturbances as predominant symptom (Table 1).

A total of 150 patients reported alterations in smell and taste during the course of the COVID-19. Isolated olfactory/smell disorders were reported in 83 cases $(55.33 \%)$ and 23 patients $(15.33 \%)$ reported isolated taste dysfunctions. 46 patients $(30.66 \%)$ reported they experienced combined disorders of both smell and taste. However, fiftyfour cases (18\%) of those having olfactory dysfunction had associated nasal obstruction (Fig. 2).

Total 129 patients reported smell alterations (86\%), among which 86 patients $(57.33 \%)$ reported complete anosmia and 43 patients $(28.66 \%)$ reported hyposmia. Sixty-nine patients $(46 \%)$ reporting taste disorders had alterations which included complete ageusia $(13.33 \%)$ and partial ageusia (32.67\%) while others in the testing population who were unable to confirm any changes in taste

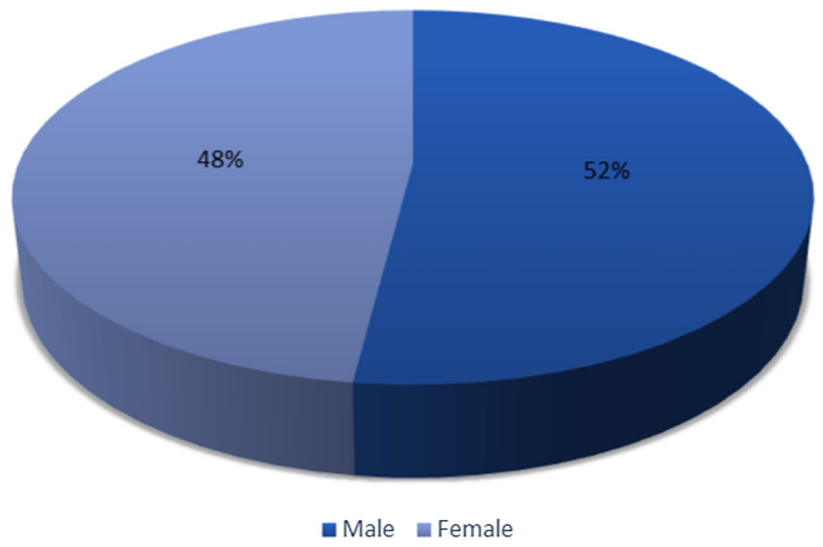

Fig. 1 Patient general characteristics 
Table 1 Clinical features

\begin{tabular}{lc}
\hline Presenting symptoms & \\
\hline Fever & $180(60 \%)$ \\
Headache & $153(51 \%)$ \\
Myalgia/Arthralgia & $144(48 \%)$ \\
Cough & $126(42 \%)$ \\
Sore throat & $117(39 \%)$ \\
Abdominal symptoms & $114(38 \%)$ \\
Smell disturbances & $105(35 \%)$ \\
Nasal obstruction/Rhinorrhoea & $54(18 \%)$ \\
Taste disturbances & $35(11.6 \%)$
\end{tabular}

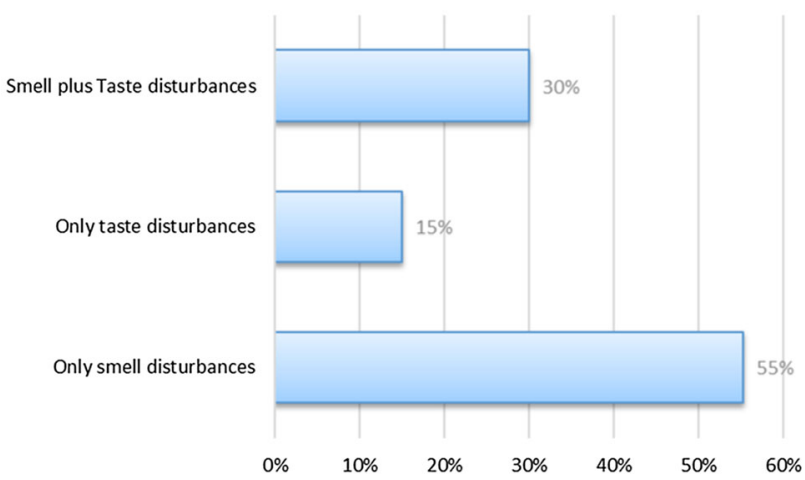

Fig. 2 Distribution of OGDs

sensation were taken as having no change in taste at all (Fig. 3).

At one month follow up, most of these patients (117 cases, $78 \%$ ) reported a complete recovery of smell, while remaining 27 cases (18\%) reported partial improvement of smell and 6 patients (4\%) reported no improvement. However all patients with taste disturbances reported complete improvement.

Among 150 patients with OGD, 66 patients (44\%) had alteration in olfaction before testing positive for COVID-

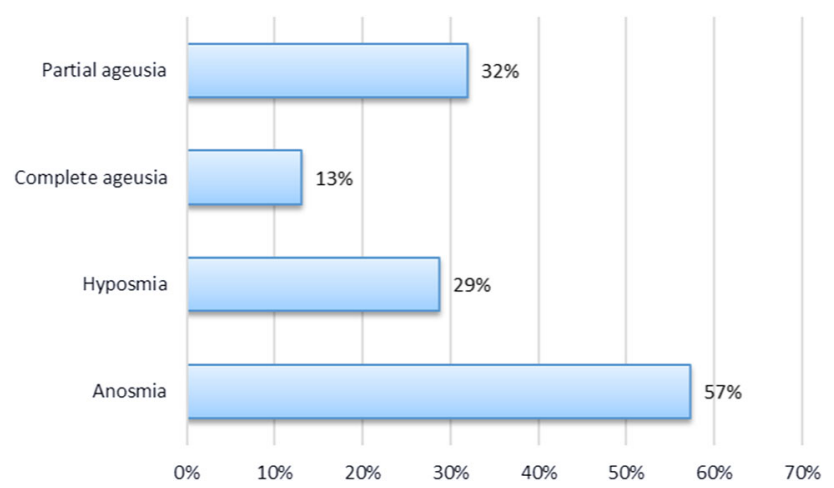

Fig. 3 Incidence of OGDs
19 disease; and 84 patients (56\%) noticed loss of smell and after testing positive for COVID-19 disease.

\section{Discussion}

Aggregating shred of evidence suggests that olfactory dysfunction is commonly found in patients of COVID-19 and has hence caught the attention of otorhinolaryngologists all over the world during COVID-19 pandemic [1].

Majority $(81 \%)$ of the patients were males with a mean age group of $37( \pm 7)$ years. This was contrary to the findings of Klopfenstein et al., who in their study had female preponderance with a mean age group of $47( \pm 16)$ years. This difference could be explained by the fact that majority of patients in our study had travelled back from other states. Patients with diabetes, neurological disorders and history of head injury were expected to have some degree of pre-existing smell or taste disorders and hence excluded from study. Similarly patients with pre-existing nasal obstruction due to allergies, polyposis or chronic sinusitis were also excluded.

The exact mechanisms underlying OGDs among patients with COVID-19 infection remain unclear. Few studies described the pathophysiological mechanisms of the olfactory and gustatory dysfunction in the SARS-CoV2 infection [16]. Available data indicate the virus has a neural spread and a cytopathic effect on the neurons. It affects mainly neurons in the cortex and hypothalamus [17]. Some authors reported three mechanisms for anosmia in COVID-19 patients: (1) local infection of support cells and vascular pericytes in the nose and olfactory bulb that may affect the function of bipolar neurons or mitral cells; (2) damage to support cells in the sensory epithelium that may indirectly influence the signaling pathway from sensory neurons to the brain; and (3) damage to sustentacular cells and Bowman's gland cells that could lead to diffuse morphological damage to the olfactory sensory epithelium and altering of smell perception [18, 19].

Anosmia is a prominent sign of SARS-CoV-2 infection [20]. Patients with COVID-19 can present a sudden onset of anosmia without any other symptoms [20, 21]. Before the onset of anosmia, other mild symptoms such as a dry cough may also be presented [22]. Several cross-sectional studies about the prevalence rate of OD in COVID-19 patients have been released, including in countries such as Italy, Spain, the United Kingdom, France, Belgium, the United States and Iran [23, 24].These surveys were typically handled through no contact methods such as online questionnaires and telephone interviews [19, 24, 25]. Incidence of OD in COVID-19 patients varied widely among these cross-sectional studies, with rates ranging from 33.9 to $68 \%$ [23-27]. The studies showed that 
individuals with smell disorders tend to have a taste disorder, suggesting a probable association between the two [23-27].

In a retrospective study by Klopfenstein et al., 54 (47\%) out of 114 confirmed COVID-19 patients presented with anosmia [28]. The study also found that patients generally developed anosmia 4.4 days after the onset of the SARSCoV-2 infection, with a duration of 8.96 days, and $98 \%$ of patients could recover within 28 days [28]. OD often accompanied by dysgeusia in COVID-19 patients [26, 29]. This study was comparable to our study as we also found the incidence of OGD to be $50 \%$ ( 150 out of 300 patients). However, time of onset of olfactory dysfunction in our study was variable contrary to the above study. This may be explained by highly variable time interval between appearance of symptoms or contact with a positive case and the date of testing. In our study we found complete recovery of olfaction in $78 \%$ of patients during first month, which is less than that found by Klopfenstein et al., (i.e. $98 \%)$.

From the above results we can say that COVID19 patients do experience olfactory dysfunction which may be anosmia or hyposmia. They may or may not be associated with gustatory dysfunction in form of ageusia or dysgeusia. Overall, we can say that anosmia and ageusia can be prevalent in COVID-19 patients but is independent of any upper respiratory infection (rhinitis/influenza).

\section{Conclusion}

Olfactory and gustatory dysfunction is prevalent in COVID-19 patients which occur independently or with other symptoms, but its pathogenesis is not well understood. Also, anosmia or hyposmia can be the sole clinical presentation without any other significant signs, hence it pushes otorhinolaryngologists to the forefront. Otolaryngologists should be hence aware of non-specific anosmia and ageusia to avoid delaying of the diagnosis of COVID19 patients.

\section{References}

1. Meng X, Deng Y, Dai Z et al (2020) COVID-19 and anosmia: a review based on up-to-date knowledge. Am J Otolaryngol 41:102581

2. Li Q, Guan X, Wu P et al (2020) Early transmission dynamics in Wuhan, China, of novel coronavirus-infected pneumonia. N Engl J Med 382:1199-1207

3. Guan WJ, Ni ZY, Hu Y et al (2020) Clinical characteristics of coronavirus disease 2019 in China. N Engl J Med 382:1708-1720

4. World Health Organization (2020) WHO Director-general's remarks at the media briefing on2019-nCoV on 11 February
2020. https://www.who.int/dg/speeches/detail/who-director-gen eral-s-remarks-at-the-media-briefing-on-2019-ncov-on-11-februa rv-2020. Accessed 3 May 2020

5. Agyeman AA, Chin KL, Landersdorfer CB et al (2020) Smell and taste dysfunction in patients with COVID-19: a systematic review and meta-analysis. J Mayo Clin Proc 95(8):1621-1631

6. Lovato A, de Filippis C, Marioni G (2020) Upper airway symptoms in coronavirus disease2019 (COVID-19). Am J Otolaryngol 41:102474

7. Lovato A, de Filippis C (2020) Clinical presentation of COVID19: a systematic review focusing on upper airway symptoms. Ear Nose Throat J 99:569-576

8. Marchese-Ragona R, Ottaviano G, Nicolai P et al (2020) Sudden hyposmia as a prevalent symptom of COVID-19 infection. medRxiv 1-4

9. Ralli M, Di Stadio A, Greco A et al (2020) Defining the burden of olfactory dysfunction in COVID-19 patients. Eur Rev Med Pharmacol Sci 24(7):3440-3441

10. Li K, Wohlford-Lenane C, Perlman S et al (2016) Middle East respiratory syndrome coronavirus causes multiple organ damage and lethal disease in mice transgenic for human dipeptidyl peptidase 4. J Infect Dis 213(5):712-722

11. Matsuo R (2000) Role of saliva in the maintenance of taste sensitivity. Crit Rev Oral Biol Med 11(2):216-229

12. Liu L, Wei Q, Alvarez X et al (2011) Epithelial cells lining salivary gland ducts are early target cells of severe acute respiratory syndrome coronavirus infection in the upper respiratory tracts of rhesus Macaques. J Virol 85(8):4025-4030

13. Zou L, Ruan F, Huang M et al (2020) SARS-CoV-2 viral load in upper respiratory specimens of infected patients. N Engl J Med 382:1177-1179

14. Sungnak W, Huang N, Bécavin C et al (2020) SARS-CoV-2 entry factors are highly expressed in nasal epithelial cells together with innate immune genes. Nat Med 26:681-687

15. Gengler I, Wang JC, Speth MM et al (2020) Sinonasal pathophysiology of SARSCoV-2 and COVID-19: a systematic review of the current evidence. Laryngoscope Investig Otolaryngol 5:354-359

16. Tanasa IA, Manciuc C, Carauleanu A et al (2020) Anosmia and ageusia associated with coronavirus infection (COVID-19)-what is known? Exp Ther Med 20:2344-2347

17. Gu J, Gong E, Zhang B et al (2005) Multiple organ infection and the pathogenesis of SARS. J Exp Med 202:415-424

18. Plasschaert LW, Žilionis R, Choo-Wing R et al (2018) A singlecell atlas of the airway epithelium reveals the CFTR-rich pulmonary ionocyte. Nature 560:377-381

19. Bihun CG, Percy DH (1995) Morphologic changes in the nasal cavity associated with sialodacryoadenitis virus infection in the Wistar rat. Vet Pathol 32:1-10

20. Heidari F, Karimi E, Firouzifar M et al (2020) Anosmia as a prominent symptom of COVID-19 infection. Rhinology 58:302-303

21. Gane SB, Kelly C, Hopkins C. Isolated sudden onset anosmia in COVID-19 infection.A novel syndrome? Rhinology 2020;58:299301.

22. Eliezer M, Hautefort C, Hamel AL et al (2020) Sudden and complete olfactory loss function as a possible symptom of COVID-19. JAMA Otolaryngol Head Neck Surg 146(7):674-675

23. Giacomelli A, Pezzati L, Conti F et al (2020) Self-reported olfactory and taste disorders in SARS-CoV-2 patients: a crosssectional study. Clin Infect Dis 71:889-890

24. Spinato G, Fabbris C, Polesel J et al (2020) Alterations in smell or taste in mildly symptomatic outpatients with SARS-CoV-2 infection. JAMA 323:2089-2090 
25. Bagheri SHR, Asghari AM, Farhadi M et al (2020) Coincidence of COVID-19 epidemic and olfactory dysfunction outbreak. medRxiv. https://doi.org/10.1101/2020.03.23.20041889

26. Menni C, Valdes A, Freydin MB et al (2020) Loss of smell and taste in combination with other symptoms is a strong predictor of COVID-19 infection. medRxiv 20048421.https://doi.org/ 10.1101/2020.04.05

27. Yan CH, Faraji F, Prajapati DP et al (2020) Association of chemosensory dysfunction and Covid-19 in patients presenting with influenza-like symptoms. Int Forum Allergy Rhinol 10(7):806-813
28. Klopfenstein T, Kadiane-Oussou NJ, Toko L et al (2020) Features of anosmia in COVID-19. Med Mal Infect. https://doi.org/10.1016/j.medmal.2020.04.006

29. Luers JC, Rokohl AC, Loreck N et al (2020) Olfactory and gustatory dysfunction in coronavirus disease 19 (COVID-19). Clin Infect Dis. https://doi.org/10.1093/cid/ciaa525

Publisher's Note Springer Nature remains neutral with regard to jurisdictional claims in published maps and institutional affiliations. 